\title{
Return time statistics for unimodal maps
}

by

\author{
H. Bruin (Groningen) and S. Vaienti (Toulon and Marseille)
}

\begin{abstract}
We prove that a non-flat S-unimodal map satisfying a weak summability condition has exponential return time statistics on intervals around a.e. point. Moreover we prove that the convergence to the entropy in the Ornstein-Weiss formula enjoys normal fluctuations.
\end{abstract}

1. Introduction and statements of the results. The subject of this paper is the asymptotic distribution of return times in dynamical systems. We consider a measure preserving ergodic dynamical system $(X, f, \mu)$. For $z \in X$, denote by $U_{z}$ a measurable set containing $z$ and by $\mu_{U_{z}}=\mu \mid U_{z} / \mu\left(U_{z}\right)$ the conditional measure on $U_{z}$. Let $\tau_{U_{z}}(x)$ be the first return of the point $x$ to $U_{z}: \tau_{U_{z}}(x)=\inf \left\{k>0 \mid f^{k}(x) \in U_{z}, x \in U_{z}\right\}$. We are interested in the distribution

$$
\mu_{U_{z}}\left(\left\{x \in U_{z} \mid \tau_{U_{z}}(x) \mu\left(U_{z}\right)>t\right\}\right)
$$

as $\mu\left(U_{z}\right) \rightarrow 0$. We refer to $[16,8,14]$ for the history and motivation of this question and for a presentation and discussion of the different techniques to solve it. Surprisingly, the limiting distribution of (1) shows a universal behavior for a wide class of dynamical systems with some degree of mixing, namely the distribution tends to $e^{-t}$. Under slightly stronger mixing conditions, one can also prove that the statistics of successive returns have a Poissonian distribution $[16,14]$. We want to stress that the convergence of the distribution (1) holds for $\mu$-a.e. $z$ and that the choice of the set $U_{z}$ is also relevant. Proofs usually require $U$ to be a cylinder set (with respect to some partition) or a ball; the use of balls is much more general and we will assume it in this paper. It is interesting that "return times to balls" recently allowed reformulating several results of thermodynamic formalism in terms

2000 Mathematics Subject Classification: Primary 37B20; Secondary 37E05, 37D25.

Key words and phrases: return time, exponential statistics.

HB was supported by the University of Toulon and the Royal Netherlands Academy of Arts and Sciences (KNAW). 
of return times statistics only (see $[15,2,31,13])$. In [31] this approach was called "thermodynamics of return times".

In [8] we advocated the technique of inducing to explain the observed universality in the distribution of return times. Let us briefly recall the main result of that paper. Assume that $X$ is a Riemannian manifold, consider an open set $\widehat{X} \subset X$ and let $\widehat{f}: \widehat{X} \rightarrow \widehat{X}$ be the first return map. We denote by $\widehat{\mu}$ the conditional measure on $\widehat{X}$, which can be proved to be $\widehat{f}$-invariant and ergodic. Let $U_{r}(z)$ be the ball of radius $r$ centered at $z \in X$ and let $\tau_{U_{r}(z)}(x)$ (resp. $\left.\widehat{\tau}_{U_{r}(z)}(x)\right)$ be the first return time to $U_{r}(z)$ for $f$ (resp. $\widehat{f}$ ). As above we denote by $\mu_{A}$ (resp. $\widehat{\mu}_{A}$ ) the conditional measure on $A$. We suppose that $(\widehat{X}, \widehat{\mu}, \widehat{f})$ has return time statistics $\widehat{g}(t)$, i.e., for $\widehat{\mu}$-a.e. $z \in \widehat{X}$, there exists $\varepsilon_{z}(r)>0$ with $\varepsilon_{z}(r) \rightarrow 0$ as $r \rightarrow 0$ such that

$$
\sup _{t \geq 0}\left|\widehat{\mu}_{U_{r}(z)}\left(\left\{x \in U_{r}(z) \mid \widehat{\tau}_{U_{r}(z)}(x)>\frac{t}{\widehat{\mu}\left(U_{r}(z)\right)}\right\}\right)-\widehat{g}(t)\right|<\varepsilon_{z}(r) .
$$

The key result of $[8]$ is that the map $\widehat{f}$ enjoys on $\widehat{X}$ the same distribution as $f$ :

THEOREM 1 (see [8]). If the function $\widehat{g}$ is continuous at 0 , then there exists $g: \mathbb{R}^{+} \rightarrow[0,1]$ such that $N:=\{x \mid g(x) \neq \widehat{g}(x)\}$ is countable and for $\mu$-a.e. $z \in \widehat{X}$ and $t \notin N$, there exists $\delta_{z, t}(r)>0$ with $\delta_{z, t}(r) \rightarrow 0$ uniformly in $t$ as $r \rightarrow 0$ such that

$$
\left|\mu_{U_{r}(z)}\left(\left\{x \in U_{r}(z) \mid \tau_{U_{r}(z)}(x)>\frac{t}{\mu\left(U_{r}(z)\right)}\right\}\right)-g(t)\right|<\delta_{z, t}(r) .
$$

This result is useful for dynamical systems that admit neighborhoods (around $\mu$-a.e. point) where the induced (i.e. first return) map is hyperbolic. The distribution in the induced system can therefore be computed by the usual techniques, quoted for example in $[16,14,8]$, and then pushed back, by Theorem 1, to the original system. We gave in [8] a few applications; in particular we proved the exponential statistics $g(t)=e^{-t}$ for $C^{3}$ interval maps of the interval such that the closure of the orbit of the critical points has zero measure.

The existence of a hyperbolic first return map around $\mu$-a.e. point is, however, unlikely. In this paper we show how, for unimodal maps, this assumption can be discarded. Instead, we impose a summability condition which is used (among other things) to guarantee the existence of an invariant probability measure $\mu$ which is absolutely continuous with respect to Lebesgue measure. This summability condition is considerably weaker than the Collet-Eckmann condition, which requires that the derivatives along the critical orbit grow exponentially fast. Hence Theorem 2 below extends the results in [29]. 
Let $f: I \rightarrow I$ be a $C^{3}$ S-unimodal map. The $\mathrm{S}$ stands for negative Schwarzian derivative, i.e., $f^{\prime \prime \prime} / f^{\prime}-\frac{3}{2}\left(f^{\prime \prime} / f^{\prime}\right)^{2}<0$ for all non-critical points. Let $c$ be the critical point, and assume that it is non-flat. This means that there exists $l \in(1, \infty)$ such that $\lim _{x \rightarrow c}|f(x)-f(c)| /|x-c|^{l}$ exists and is positive. Denote $f^{i}(c)$ by $c_{i}$. In Section 2 we will prove the following theorem:

ThEOREM 2. Let $f$ be a non-flat $C^{3}$ S-unimodal map satisfying

$$
\sum_{n \geq 1}\left|D f^{n-1}\left(c_{1}\right)\right|^{-1 / l}<\infty
$$

Let $\mu$ be the invariant measure which is absolutely continuous with respect to Lebesgue measure. Then $f$ has exponential return time statistics on intervals, i.e., for $\mu$-a.e. $z$,

$$
\mu_{U}\left(\left\{y \mid \tau_{U}(y)>t / \mu(U)\right\}\right) \rightarrow e^{-t} \quad \text { as } \mu(U) \rightarrow 0,
$$

where $U$ are intervals around $z$.

The summability condition (2) is the same as was used by Nowicki \& van Strien [27] to show the existence of an absolutely continuous invariant probability measure $\mu$. Clearly (2) is more inclusive than the Collet-Eckmann condition which was used in comparable results on return statistics $[3,9]$. In particular, Theorem 2 applies to systems where the decay of correlations is not exponentially fast (cf. [26]).

As a second main result, we will prove the log-normal fluctuations for the entropy given by the Ornstein-Weiss formula. Given a generating partition $\mathcal{C}$ and corresponding cylinder sets $C_{n}(x)$, this formula says that the first return of $\mu$-a.e. $x$ grows like $e^{n h_{\mu}}$, where $h_{\mu}$ is the measure theoretic entropy of $\mu$. The distribution of the process $\left(\log \tau_{C_{n}(x)}(x)-n h_{\mu}\right) / \sqrt{n}$, suitably normalized, will converge in law to the Gauss zero-one law.

In Section 3, we will show that this applies to unimodal maps and $\mathcal{C}=$ $\left\{\left[c_{2}, c\right],\left[c, c_{1}\right]\right\}$, the partition into the two monotonicity intervals. To the absolutely continuous invariant measure $\mu$ we associate the variance $\sigma_{\mu}$ :

$$
\sigma_{\mu}^{2}=\sigma_{\mu}^{2}(\varphi)=\int \varphi^{2} d \mu-\left(\int \varphi d \mu\right)^{2}+2 \sum_{n=1}^{\infty}\left[\int \varphi\left(f^{n}\right) \varphi d \mu-\left(\int \varphi d \mu\right)^{2}\right]
$$

where $\varphi=\log \left|f^{\prime}\right|-\int \log \left|f^{\prime}\right| d \mu$.

TheOREm 3. Let $f$ be a non-flat $C^{3}$ S-unimodal interval map. Assume that $\left|D f^{n}\left(c_{1}\right)\right| \geq C n^{\tau}$ for some $\tau>4 l-3$ and $C>0$. If $\sigma_{\mu}^{2}>0$, then

$$
\mu\left(\left\{x \in I \mid \frac{\log \tau_{C_{n}(x)}(x)-n h_{\mu}}{\sigma_{\mu} \sqrt{n}}>u\right\}\right) \Rightarrow \frac{1}{\sqrt{2 \pi}} \int_{u}^{\infty} e^{-x^{2} / 2} d x .
$$


The most difficult part in the proof will be the verification of the Central Limit Theorem for the potential $\varphi$ (see Theorem 4). We think that this is interesting enough to be stated separately.

THEOREM 4. Under the assumptions of Theorem 3, $\varphi$ satisfies the Central Limit Theorem.

\section{The exponential statistics}

2.1. The Hofbauer tower. Let us come back to the unimodal map $f$ : $I \rightarrow I$ defined in the introduction. If we denote $f^{i}(c)$ by $c_{i}$, we can rescale, without loss of generality, $f$ so that $I=\left[c_{2}, c_{1}\right]$. Condition (2) implies that $c$ is not attracted to a stable periodic orbit, nor is $f$ infinitely renormalizable.

We presently describe the canonical Markov extension (Hofbauer tower) of $f$. Let $D_{2}=\left[c_{2}, c_{1}\right]$ and

$$
D_{k+1}= \begin{cases}f\left(D_{k}\right) & \text { if } c \notin D_{k}, \\ {\left[c_{k+1}, c_{1}\right]} & \text { if } c \in D_{k} .\end{cases}
$$

A short induction argument shows that $D_{k}=\left[c_{k}, c_{\beta(k)}\right]$ or $D_{k}=\left[c_{\beta(k)}, c_{k}\right]$, where $\beta(k)=k-\max \left\{i<k \mid D_{i} \ni c\right\}$. See [4] for a proof and additional information. The Hofbauer tower is the disjoint union $\widehat{I}:=\bigsqcup_{k \geq 2} D_{k}$. We can define the following map $\widehat{g}$ :

$$
\widehat{g}\left(x \in D_{k}\right)=f(x) \in \begin{cases}D_{k+1} & \text { if } c \notin\left[c_{k}, x\right], \\ D_{\beta(k)+1} & \text { if } c \in\left[c_{k}, x\right] .\end{cases}
$$

By construction, $(\widehat{I}, \widehat{g})$ is a Markov map, in the sense that the image of each level $D_{k}$ is precisely the union of some levels $D_{i}$. Let $\pi: \widehat{I} \rightarrow I$ be the natural projection. By construction $(I, f)$ is a factor of $(\widehat{I}, \widehat{g}): \pi \circ \widehat{g}=f \circ \pi$. Keller's result [17] states that the measure $\mu$ can be lifted to a measure $\widehat{\mu}$ on $\widehat{I}$ which is $\widehat{g}$-invariant, absolutely continuous and satisfies $\mu=\widehat{\mu} \circ \pi^{-1}$. In short, $\widehat{\mu}$ is constructed as the vague limit of the following sequence:

$$
\begin{aligned}
\widehat{\mu}_{1} \mid D_{2} & =\text { normalized Lebesgue, } \quad \widehat{\mu}_{1} \mid D_{k} \equiv 0 \quad \text { for } k \geq 3, \\
\widehat{\mu}_{n} & =\widehat{\mu}_{n-1} \circ \widehat{g}^{-1} .
\end{aligned}
$$

Several of our estimates are based on versions of the Koebe Lemma. We recall it in the form that we use. Originally this lemma is proved under the assumption that $f$ has negative Schwarzian derivative. Recent work of Kozlovski shows that the $C^{3}$ assumption is sufficient (see [24, Section IV.3] and [21]), but the focus of our results would not justify more complicated proofs avoiding the negative Schwarzian assumption.

Lemma 1. Let $g:(a, b) \rightarrow \mathbb{R}$ be a monotone $C^{3}$ map with negative Schwarzian derivative. Then for any $\delta>0$, there is a Koebe constant $K=$ $K(\delta)=((1+\delta) / \delta)^{2}$ such that the following hold: 
- (Koebe Principle) If $x \in(a, b)$ is such that

$$
\frac{\min \{|g(b)-g(x)|,|g(x)-g(a)|\}}{|g(b)-g(a)|} \geq \delta
$$

then

$$
\frac{1}{K} \frac{|g(b)-g(a)|}{|b-a|} \leq\left|g^{\prime}(x)\right| \leq K \frac{|g(b)-g(a)|}{|b-a|} .
$$

- (Macroscopic Koebe Principle) Under the same hypothesis,

$$
\frac{\min \{|b-x|,|x-a|\}}{|b-a|} \geq \frac{1}{K^{2}} \text {. }
$$

Lemma 2. Assume that $f$ is conservative with respect to Lebesgue measure. There exists $\delta>0$ such that for Lebesgue a.e. $x \in I$, there exist sequences of intervals $I_{n} \supset J_{n} \ni x$ satisfying:

- both components of $I_{n} \backslash J_{n}$ have length $\geq \delta\left|I_{n}\right|$;

- $\left|I_{n}\right| \rightarrow 0$

- $f^{i}\left(\partial J_{n}\right) \cap I_{n}^{\circ}=\emptyset$ for all $i \geq 1$. (Here $I_{n}^{\circ}$ denotes the interior.)

The sets $J_{n}$ (or rather certain lifts of them) will play the role of $\widehat{X}$ from Theorem 1 . The property $\operatorname{orb}\left(\partial J_{n}\right) \cap J_{n}=\emptyset$ implies that any branch $f^{s}: H \subset J_{n} \rightarrow J_{n}$ of the first return map to $J_{n}$ (i.e., $H$ is a maximal interval on which this first return map is continuous) satisfies $f^{s}(\partial H) \subset \partial J_{n}$. In particular, if $f^{s} \mid H$ is monotone, then $f^{s}(H)=J_{n}$. Most of these branches are extendible to an onto branch $f^{s}: H^{\prime} \rightarrow I_{n}$, and then the Koebe Principle implies that the distortion of $f^{s} \mid H$ is uniformly bounded (by $K(\delta)$ ).

Proof of Lemma 2. The proof depends on a result of Martens [23, Lemma 4.2 ] valid for conservative maps. It states that there exist symmetric intervals $V, U$ around $c$, with $V$ compactly contained in $U$, such that both $f^{i}(\partial V)$ and $f^{i}(\partial U)$ are disjoint from the interior of $U$ for all $i \geq 1$. Furthermore, for Lebesgue a.e. $x$ there exists an integer sequence $k_{n} \rightarrow \infty$ and intervals $I_{n} \supset J_{n} \ni x$ such that $f^{k_{n}}$ maps $I_{n}$ monotonically onto $U$ and $J_{n}$ onto $V$. By the macroscopic Koebe Principle, there exists $\delta>0$, depending only on $V$ and $U$, such that both components of $I_{n} \backslash J_{n}$ have length $\geq \delta\left|I_{n}\right|$. Moreover, if $f^{i}\left(\partial J_{n}\right) \cap I_{n}^{\circ} \neq \emptyset$ for some $i>0$, then $f^{k_{n}+i}\left(\partial J_{n}\right)=f^{i}(\partial V)$ intersects $f^{k_{n}}\left(I_{n}^{\circ}\right)=U^{\circ}$, contradicting the properties of $V$. Finally, due to the absence of wandering intervals (see [24, p. 267]), $\left|I_{n}\right| \rightarrow 0$ as $n \rightarrow \infty$. Hence the $I_{n}$ and $J_{n}$ have all the asserted properties.

Given $I_{n} \supset J_{n}$, let $\widehat{J}_{n}=\bigsqcup\left\{D_{k} \cap J_{n} \mid D_{k} \supset I_{n}\right\} \subset \widehat{I}$. Note that, in general, $\pi^{-1}\left(J_{n}\right)$ strictly contains $\widehat{J}_{n}$. Only if $x \notin \overline{\operatorname{orb}(c)}$, the two sets are equal for $n$ sufficiently large. Define $\widehat{F}_{n}: \widehat{J}_{n} \rightarrow \widehat{J}_{n}$ to be the first return map. It is known (see [17, Theorem 1(3)]) that $(\widehat{I}, \widehat{g})$ is ergodic and conservative. Therefore $\widehat{F}_{n}$ is defined $\widehat{\mu}$-a.e. on $\widehat{J}_{n}$. By the Markov property of the Hofbauer 
tower and the construction of $J_{n}$ it follows that $\widehat{F}_{n}$ is an extendible Markov map in the sense that if $y \in \widehat{J}_{n}$ and $\widehat{F}_{n} \mid\{y\}=\widehat{g}^{i}$, then there exists an interval $V \ni y$ such that $\widehat{g}^{i}$ maps $V$ continuously and monotonically onto an interval $W$ such that $\pi(W)=I_{n}$. These ideas were discussed in detail in [5]. It follows by the Koebe Principle that all branches of all iterates of $\widehat{F}_{n}$ have the same distortion bounds. Moreover, there exists $k_{0}$ such that $\inf _{k \geq k_{0}} \inf _{y \in \widehat{J}_{n}}\left|D \widehat{F}_{n}^{k}(y)\right|>1$.

Lemma 3. Let $U$ be any subinterval of $J_{n}$ and $\widehat{U}_{n}:=\pi^{-1}(U) \cap \widehat{J}_{n}$. Then the system $\left(\widehat{J}_{n}, \widehat{\mu}_{\widehat{J}_{n}}, \widehat{F}_{n}\right)$ has exponential return time statistics on $\widehat{U}_{n}$.

Proof. The map $\widehat{F}_{n}$ corresponds to a jump transformation $F_{n}$ on $J_{n}$, defined by $F_{n}(y)=\pi \circ \widehat{F}_{n} \circ \pi^{-1}(y)$. The above described properties of $\widehat{F}_{n}$ show that $F_{n}$ is well defined. In fact $F_{n}(y)=f^{\widetilde{\tau}(y)}(y)$, where $\widetilde{\tau}(y)$ is the first $n$-decent return to $J_{n}$, defined as

$\widetilde{\tau}(y)=\widetilde{\tau}_{U}(y)=\min \left\{i>0 \mid f^{i}(y) \in U\right.$ and there exists an interval $K \ni y$ such that $f^{i}: K \rightarrow I_{n}$ is monotone onto $\}$.

As was shown in [5], $\widetilde{\tau}(y)=\widehat{\tau}(\widehat{y})$ for any $\widehat{y} \in \widehat{J}_{n}$ and $\widehat{\tau}$ the first return time to $\widehat{J}_{n}$. The map $F_{n}$ is a Rychlik map as defined in [8]. The results of that paper yield the exponential return statistics.

2.2. Proof of Theorem 2. Let us first collect some facts on the invariant density $h(y)=d \mu(y) / d y$ of $\mu$. Define $\delta_{k}=\left|D f^{k-1}\left(c_{1}\right)\right|^{-1 / l}$. It was shown by Nowicki [25] that for some constant $C>0$,

$$
h(y) \leq C \sum_{k \geq 1} \delta_{k}\left|y-c_{k}\right|^{-(1-1 / l)} .
$$

In particular

$$
\sum_{k} \delta_{k}\left|c_{k}-x\right|^{1 / l-1}<\infty \quad \text { for } \mu \text {-a.e. } x \text {. }
$$

If, in addition, $f$ satisfies the Collet-Eckmann condition (i.e., $\delta_{k} \rightarrow 0$ exponentially), then more precise estimates are known [32, 19]. Keller \& Nowicki showed that $h(y)=\psi(y) \sum_{k \geq 1} \delta_{k}\left|y-c_{k}\right|^{-(1-1 / l)}$, where $\psi$ has bounded variation. For our purpose we only need the upper bound in (5). It immediately follows that the density $h$ is finite at $c$. Indeed, the Chain Rule and non-flatness give

$$
\left|D f^{k}\left(c_{1}\right)\right|=\left|D f^{k-1}\left(c_{1}\right)\right|\left|D f\left(c_{k}\right)\right|=\mathcal{O}(l)\left|D f^{k-1}\left(c_{1}\right)\right|\left|c-c_{k}\right|^{l-1} .
$$

Therefore

$$
\begin{aligned}
h(c) & \leq C \sum_{k}\left|D f^{k-1}\left(c_{1}\right)\right|^{-1 / l}\left|c-c_{k}\right|^{1-1 / l} \\
& \leq \mathcal{O}(C l) \sum_{k}\left|D f^{k}\left(c_{1}\right)\right|^{-1 / l}<\infty .
\end{aligned}
$$


We want to consider "intermediate" first return maps to $J_{n}$, where $J_{n}$ are neighborhoods of $x$ as introduced in Lemma 2, and eventually we take limits $n \rightarrow \infty$, i.e., $\left|J_{n}\right| \rightarrow 0$. Assume $x \notin \operatorname{orb}(c)$. Since endpoints of level $D_{k}$ belong to orb $(c)$, every level $D_{k}$ that contains $x$ also contains $I_{n}$ for $n$ large. Therefore more returns to a small neighborhood $U, x \in U \subset I_{n}$, will be $n$-decent if $n$ gets larger (so the intervals $U$ we can consider depend on $n$ ). We will need a precise statement on the proportion of decent branches for the return map to $U$.

Lemma 4. For any $x$ satisfying the assertion of Lemma 2 and (6),

$$
\lim _{n \rightarrow \infty} \sup _{x \in U \subset J_{n}} \mu_{U}\left(\left\{y \mid \tau_{U}(y) \text { is not n-decent }\right\}\right)=0 .
$$

Proof. As in Lemma 3, set $\widehat{U}_{n}:=\pi^{-1}(U) \cap \widehat{J}_{n}$. Define $\widehat{h}(y)=d \widehat{\mu}(y) / d y$ and let $\widehat{h}_{k}=\widehat{h} \mid D_{k}$ be the densities on levels of $\widehat{I}$. Clearly $h=\sum_{k} \widehat{h}_{k}$. Moreover, $\widehat{h}$ is a fixed point of the Perron-Frobenius operator on $\widehat{I}$ and the points $c \in D_{k}$ are the only critical points of $\widehat{g}$.

Due to the Markov property of $(\widehat{I}, \widehat{g}), \widehat{h}_{k}$ has only two singularities, namely at the endpoints $c_{k}$ and $c_{\beta(k)}$. More precisely, letting $a_{k}=\widehat{h}_{k}(c)$ when $c \in D_{k}$ and $a_{k}=0$ otherwise, we have, for $y \in D_{k}=\left[c_{k}, c_{\beta(k)}\right]$,

$$
\widehat{h}_{k}(y) \leq C\left(\delta_{k}\left|y-c_{k}\right|^{-(1-1 / l)}+a_{k-\beta(k)} \delta_{\beta(k)}\left|y-c_{\beta(k)}\right|^{-(1-1 / l)}\right) .
$$

The first term is obvious because $\widehat{h}_{k} \leq h$; the second term arises because the endpoint $c_{\beta(k)}$ is the image of $c \in D_{k-\beta(k)}$ under $\widehat{g}^{k-\beta(k)}$.

Now let $x$ satisfy the conclusion of Lemma 2 (so that the sets $I_{n} \supset J_{n} \ni x$ are well defined), and also (6). Take an interval $U=\left(u_{0}, u_{1}\right)$ such that $x \in U \subset J_{n}$. Let $\widehat{\Phi}: \pi^{-1}(U) \rightarrow \pi^{-1}(U)$ be the first return map. By $\widehat{\Phi}$ invariance of $\widehat{\mu}_{\pi^{-1}(U)}$ we find $\mu\left(\left\{y \in U \mid \tau_{U}\right.\right.$ is not $n$-decent $\left.\}\right)=\widehat{\mu}\left(\widehat{\Phi}^{-1}\left(\pi^{-1}(U) \backslash \widehat{U}_{n}\right)\right)=\widehat{\mu}\left(\pi^{-1}(U) \backslash \widehat{U}_{n}\right)$.

We know (see $[18,6])$ that $h$ is bounded away from 0 , say $h_{0}=\inf _{\operatorname{supp}(\mu)} h>0$. Then we can integrate

$$
\begin{aligned}
& \frac{\widehat{\mu}\left(\pi^{-1}(U) \cap D_{k}\right)}{\mu(U)} \leq \frac{\widehat{\mu}\left(\pi^{-1}(U) \cap D_{k}\right)}{h_{0}\left|u_{1}-u_{0}\right|} \\
& \leq \frac{C}{h_{0}\left|u_{1}-u_{0}\right|} \int_{u_{0}}^{u_{1}}\left(\delta_{k}\left|c_{k}-y\right|^{1 / l-1}+\delta_{\beta(k)} a_{k-\beta(k)}\left|c_{\beta(k)}-y\right|^{1 / l-1}\right) d y \\
& \leq \frac{C l}{h_{0}}\left(\delta_{k}\left|c_{k}-x\right|^{1 / l-1}+\delta_{\beta(k)} a_{k-\beta(k)}\left|c_{\beta(k)}-x\right|^{1 / l-1}\right) .
\end{aligned}
$$

As $x \neq c_{k}$ for any $k \geq 0$, there exists $N=N(n)$ such that all returns to 
levels $D_{k}$ with $k \leq N$ are $n$-decent, and $N(n) \rightarrow \infty$ as $n \rightarrow \infty$. Therefore

$$
\begin{aligned}
& \frac{\mu\left(\left\{y \in U \mid \tau_{U} \text { is not } n \text {-decent }\right\}\right)}{\mu(U)} \\
& \quad \leq \frac{C l}{h_{0}} \sum_{k \geq N}\left(\delta_{k}\left|c_{k}-x\right|^{1 / l-1}+\delta_{\beta(k)} a_{k-\beta(k)}\left|c_{\beta(k)}-x\right|^{1 / l-1}\right) .
\end{aligned}
$$

As $\sum_{k} a_{k}=h(c)<\infty$ and because of $(6)$, both $\delta_{k}\left|c_{k}-x\right|^{1 / l-1}$ and $a_{k}$ are summable. Therefore

$$
\lim _{n \rightarrow \infty} \frac{\mu\left(\left\{y \in U \mid \tau_{U} \text { is not } n \text {-decent }\right\}\right)}{\mu(U)}=0,
$$

uniformly in $U$.

Now we are ready to prove the main theorem.

Proof of Theorem 2. Let $\alpha_{n}=\sup _{x \in U \subset J_{n}} \widehat{\mu}\left(\widehat{U}_{n}\right) / \mu(U)$. As we have seen in Lemma $4, \lim _{n} \alpha_{n}=1$. Because $f \circ \pi=\pi \circ \widehat{g}$ we have

$$
\mu_{U}\left(\left\{y \mid \tau_{U}(y)>t / \mu(U)\right\}\right)=\widehat{\mu}_{\pi^{-1}(U)}\left(\left\{\widehat{y} \mid \widehat{\tau}_{\pi^{-1}(U)}(\widehat{y})>t / \mu(U)\right\}\right) .
$$

The right hand side can be rewritten as a sum of three terms:

$$
\begin{aligned}
\text { r.h.s. } \leq & \widehat{\mu}_{\pi^{-1}(U)}\left(\pi^{-1}(U) \backslash \widehat{U}_{n}\right) \\
& +\widehat{\mu}_{\pi^{-1}(U)}\left(\left\{\widehat{y} \in \widehat{U}_{n} \mid \widehat{\tau}_{\widehat{U}_{n}}(\widehat{y})>t / \mu(U)\right\}\right) \\
& +\widehat{\mu}_{\pi^{-1}(U)}\left(\left\{\widehat{y} \in \widehat{U}_{n} \mid \widehat{\tau}_{\widehat{U}_{n}}(\widehat{y})>\tau_{\pi^{-1}(U)}(\widehat{y})\right\}\right) \\
= & I+I I+I I I .
\end{aligned}
$$

We have the estimate

$$
I=\frac{\widehat{\mu}\left(\pi^{-1}(U) \backslash \widehat{U}_{n}\right)}{\widehat{\mu}\left(\pi^{-1}(U)\right)}=\frac{\widehat{\mu}\left(\pi^{-1}(U) \backslash \widehat{U}_{n}\right)}{\mu(U)} \leq 1-\alpha_{n} \rightarrow 0 .
$$

Next

$$
I I=\alpha_{n} \widehat{\mu}_{\widehat{U}_{n}}\left(\left\{\widehat{y} \mid \widehat{\tau}_{\widehat{U}_{n}}(\widehat{y})>t / \mu(U)\right\}\right)=\alpha_{n} \widehat{\mu}_{\widehat{U}_{n}}\left(\left\{\widehat{y} \mid \widehat{\tau}_{\widehat{U}_{n}}(\widehat{y})>\widetilde{t} / \widehat{\mu}\left(\widehat{U}_{n}\right)\right\}\right)
$$

for $\tilde{t}=t \alpha_{n}$. The main result of [8] says that the return statistics of an induced transformation coincides with the return statistics of the original system. In this case, it means that the system $(\widehat{I}, \widehat{\mu}, \widehat{g})$ has the same return statistics on $\widehat{U}_{n}$ as the induced system $\left(\widehat{J}_{n}, \widehat{\mu}_{\widehat{J}_{n}}, \widehat{F}_{n}\right)$. By Lemma 3 , these statistics have the exponential distribution. Hence $I I$ tends to $\alpha_{n} e^{-\widetilde{t}}$ as $\mu(U) \rightarrow 0$, and thus to $e^{-t}$ as $n \rightarrow \infty$. The third term satisfies

$$
\begin{aligned}
I I I & =\widehat{\mu}_{\pi^{-1}(U)}\left(\widehat{\Phi}^{-1}\left(\pi^{-1}(U) \backslash \widehat{U}_{n}\right) \cap \widehat{U}_{n}\right) \\
& \leq \widehat{\mu}_{\pi^{-1}(U)}\left(\pi^{-1}(U) \backslash \widehat{U}_{n}\right)=I \rightarrow 0
\end{aligned}
$$


as $n \rightarrow \infty$. This gives the required upper bound for $\mu_{U}\left(\left\{y \mid \tau_{U}(y)>\right.\right.$ $t / \mu(U)\})$. Now for the lower bound:

$$
\begin{aligned}
\text { r.h.s. } \geq & \widehat{\mu}_{\pi^{-1}(U)}\left(\left\{\widehat{y} \in \widehat{U}_{n} \mid \widehat{\tau}_{\widehat{U}_{n}}(\widehat{y})>t / \mu(U)\right\}\right) \\
& -\widehat{\mu}_{\pi^{-1}(U)}\left(\left\{\widehat{y} \in \widehat{U}_{n} \mid \tau_{\widehat{U}_{n}}(\widehat{y})>\tau_{\pi^{-1}(U)}(\widehat{y})\right\}\right) \\
= & I I-I I I .
\end{aligned}
$$

The above arguments show that this also tends to $e^{-t}$ as $\mu(U) \rightarrow 0$ and $n \rightarrow \infty$.

REMARK 1. It has been shown in [16] that the $e^{-t}$ statistics for local return times imply the analogous statistics for entrance times. Therefore for our unimodal maps we get the additional interesting result:

$$
\left|\mu\left(\left\{x \in X \mid \tau_{U_{r}(z)}(x)>t / \mu\left(U_{r}(z)\right)\right\}\right)-e^{-t}\right| \rightarrow 0
$$

as $r \rightarrow 0$ and for $\mu$-almost all $z \in X$.

REMARK 2. A lot of work has recently been devoted to the estimation of the error of the limiting distribution $e^{-t}$ (see for example [16, 14, 30, 11, 1, 29]). This error is related to the rate of mixing of the systems and sometimes to the degree of hyperbolicity [16]. In particular for some Gibbsian sources, the error can be obtained by looking at the asymptotic behavior of the first return of the set $U_{z}$ to itself [30]. An interesting improvement of our techniques would be to push back the error term from the induced system (where it can very often be computed) to the original one; this will probably require a more constructive way of inducing.

REMARK 3. As already pointed out in [8], the techniques of inducing can be easily adapted to the statistics of successive return times. This means, with the obvious change of notation, studying the asymptotic distribution of the quantity

$$
\mu_{U_{r}(z)}\left(\left\{x \in U_{r}(z) \mid \tau_{U_{r}}^{k-1} \mu\left(U_{r}\right) \leq t<\tau_{U_{r}}^{k} \mu\left(U_{r}\right)\right\}\right)
$$

as $r \rightarrow 0$, where $\tau_{A}^{k}$ is the $k$ th return time to $A$. Under additional mixing conditions ( $\alpha$-mixing) one can prove that the limiting distribution is the Poisson law: $\left(t^{k} / k !\right) e^{-t}$ for $\mu$-a.e. $z$.

3. Fluctuations of entropy. One of the most interesting consequences of the exponential statistics for return times is the behavior of the fluctuations in the convergence to the measure theoretic entropy as given by the Ornstein-Weiss formula (see [10] and [20] for the first works in this direction). Let $C_{n}(x)$ be the unique element of $\mathcal{C}_{n}=\bigvee_{i=1}^{n} T^{-(i-1)} \mathcal{C}$ which contains the point $x \in X$, where $\mathcal{C}$ is a finite generating partition of our ergodic system $(X, f, \mu)$. Assume that the sum $H_{\mu}(\mathcal{C})=-\sum_{C \in \mathcal{C}} \mu(C) \log \mu(C)$ is 
finite. The Ornstein-Weiss Theorem [28] asserts that

$$
\lim _{n \rightarrow \infty} \frac{1}{n} \log \tau_{C_{n}(x)}(x)=h_{\mu} \quad \text { for } \mu \text {-a.e. } x \in X,
$$

where $h_{\mu}$ is the measure theoretical entropy of $\mu$. We will define in a moment a convenient partition for our class of unimodal maps. Assume that the variance $\sigma_{\mu}^{2}(\varphi)$ as defined in (3) is positive. We are interested in showing the following convergence in distribution as $n \rightarrow \infty$ :

$$
\mu\left(\left\{x \in X \mid \frac{\log \tau_{C_{n}(x)}(x)-n h_{\mu}}{\sigma_{\mu} \sqrt{n}}>u\right\}\right) \Rightarrow \frac{1}{\sqrt{2 \pi}} \int_{u}^{\infty} e^{-x^{2} / 2} d x .
$$

Theorem 5 uses a sufficient condition due to Saussol [30] to prove the convergence (4). Saussol's result is however much more general since it states the equivalence of the fluctuations to the entropy according to the OrnsteinWeiss and Shannon-McMillan formulas provided the $e^{-t}$ law holds for local return times uniformly in $t$.

Theorem 5 (see [30]). Define the error of the asymptotic distribution of the return times into cylinders as

$$
E_{\mu}\left(C_{n}(x)\right)=\sup _{t \geq 0}\left|\mu_{C_{n}(x)}\left(\left\{x \in C_{n}(x) \mid \tau_{C_{n}(x)} \mu\left(C_{n}(x)\right)>t\right\}\right)-e^{-t}\right| .
$$

Suppose that:

(i) $E_{\mu}\left(C_{n}(x)\right) \rightarrow 0$ for $\mu$-a.e. $x$ as $\mu\left(C_{n}(x)\right) \rightarrow 0$;

(ii) the fluctuations in the Shannon-McMillan Theorem are log-normal, i.e.

$$
\mu\left(\left\{x \in X \mid \frac{-\log \mu\left(C_{n}(x)\right)-n h_{\mu}}{\sigma_{\mu} \sqrt{n}}>u\right\}\right) \Rightarrow \frac{1}{\sqrt{2 \pi}} \int_{u}^{\infty} e^{-x^{2} / 2} d x
$$

where $0<\sigma_{\mu}<\infty$.

Then the limit (4) follows.

3.1. Proof of Theorem 3. We verify the assumptions of the preceding theorem. Assumption (i) is just the content of the exponential statistics established in Section 2. It remains to prove assumption (ii). This will be achieved in two steps whose details will be given below:

- The invariant measure $\mu$ enjoys a weak-Gibbs property. This means that for $\mu$-a.e. $x$ we have

$$
\mu\left(C_{n}(x)\right) \in\left[c_{1}(n)\left|D f^{n}(x)\right|^{-1}, c_{2}(n)\left|D f^{n}(x)\right|^{-1}\right],
$$

where $c_{1}(n)$ and $c_{2}(n)$ decrease (resp. increase) subexponentially in $n$. To be precise, we need

$$
e^{-n^{\alpha}} \leq c_{1}(n) \leq c_{2}(n) \leq e^{n^{\alpha}}
$$


for some $0<\alpha<1 / 2$. (We refer to [10] and [15] for the details on Gibbs sources and weak-Gibbs measures.) This allows us to replace the measure of a cylinder of length $n$ with the sum $S_{n}:=\sum_{l=0}^{n-1} \log \left|D f\left(f^{l}\right)\right|$. Then the Cesàro mean converges to the positive Lyapunov exponent of the measure $\mu$ which is also equal to the measure theoretic entropy $h_{\mu}$ (see [22]): $h_{\mu}=$ $\int \log \left|f^{\prime}\right| d \mu$. More precisely, we have the upper bound

$$
\begin{aligned}
\mu(\{x & \left.\left.\in X \mid \frac{-\log \mu\left(C_{n}(x)\right)-n h_{\mu}}{\sigma_{\mu} \sqrt{n}}>u\right\}\right) \\
& \leq \mu\left(\left\{x \in X \mid \frac{\log \left|D f^{n}(x)\right|-n h_{\mu}}{\sigma_{\mu} \sqrt{n}}>u+\frac{\log c_{1}(n)}{\sigma_{\mu} \sqrt{n}}\right\}\right) \\
& \leq \mu\left(\left\{x \in X \mid \frac{\sum_{i=0}^{n-1} \log \left|D f\left(f^{i}(x)\right)\right|-n h_{\mu}}{\sigma_{\mu} \sqrt{n}}>u-\frac{n^{\alpha}}{\sigma_{\mu} \sqrt{n}}\right\}\right) \\
& \leq \mu\left(\left\{x \mid \frac{\sum_{i=0}^{n-1} \log \left|D f\left(f^{i}(x)\right)\right|-n h_{\mu}}{\sigma_{\mu} \sqrt{n}}>u-\delta\right\}\right) \\
& =\mu\left(\left\{x \mid \frac{\sum_{i=0}^{n-1} \varphi\left(f^{i}(x)\right)}{\sigma_{\mu} \sqrt{n}}>u-\delta\right\}\right),
\end{aligned}
$$

where $\delta$ is any positive number greater than $n^{\alpha} /\left(\sigma_{\mu} \sqrt{n}\right)$. Since $\alpha<1 / 2$, we can take $\delta \rightarrow 0$ as $n \rightarrow \infty$. Similar estimates hold for the lower bound.

- Suppose now that the Central Limit Theorem holds for the potential $\varphi=\log \left|f^{\prime}\right|-\int \log \left|f^{\prime}\right| d \mu$ with variance $\sigma_{\mu}>0$. In this case we can continue the lower bound of the above item as

$$
\liminf _{n \rightarrow \infty} \mu\left(\left\{x \mid \mu\left(C_{n}(x)\right)<e^{-n h_{\mu}-\sigma_{\mu} u \sqrt{n}}\right\}\right) \geq \frac{1}{\sqrt{2 \pi}} \int_{u-\delta}^{\infty} e^{-x^{2} / 2} d x,
$$

which gives the desired result for the lower bound as $\delta \rightarrow 0$.

The proofs of the weak-Gibbs property of $\mu$ and the Central Limit Theorem are given in the next subsections.

The weak-Gibbs property and the Central Limit Theorem for the potential $\varphi$ are interesting in their own right, as they allow application of thermodynamic formalism to our unimodal maps and they establish finer fluctuation behavior. We think for example of the large deviations property for the random processes $\log \mu\left(C_{n}(x)\right)$ and $\log \tau_{C_{n}(x)}(x)$. (See [15] for related results in the case of $(\varphi-f)$-mixing systems.)

3.2. The weak-Gibbs property. Let $\mathcal{C}=\left\{\left[c_{2}, c\right],\left[c, c_{1}\right]\right\}$ be the partition into the two monotonicity intervals of $f$. In the absence of attracting periodic points, this partition is generating. Let as before $\mathcal{C}_{n}=\mathcal{C} \vee f^{-1}(\mathcal{C}) \vee \ldots \vee$ $f^{-(n-1)}(\mathcal{C})$, and let $C_{n}(x)$ be the $n$-cylinder containing $x$. 
Lemma 5. Suppose $f$ satisfies condition (2), and let $\mu$ be its absolutely continuous invariant measure. Then for any $\varepsilon>0$ and $\mu$-a.e. $x$ there exists $n_{0}$ such that

$$
\frac{b}{n^{3(l+1)}} \leq \frac{b}{n^{2(l+1)}}\left|f^{n}\left(C_{n}(x)\right)\right| \leq \mu\left(C_{n}(x)\right)\left|D f^{n}(x)\right| \leq n^{2(1+\varepsilon)}
$$

for all $n \geq n_{0}$ and $b=\frac{1}{2} \inf _{\operatorname{supp}(\mu)} d \mu(y) / d y>0$.

Proof. The upper bound: We have the invariant measure $\mu$ and Lebesgue measure $m$. The arguments use the Borel-Cantelli Lemma applied to $m$. Let $W_{n}$ be the collection of $n$-cylinders $C$ such that $\mu(C)>n^{1+\varepsilon} m(C)$. Write $A_{n}=\bigcup_{C \in W_{n}} C$. Since $\mu$ is a probability measure, $1 \geq \sum_{C \in W_{n}} \mu(C) \geq$ $n^{1+\varepsilon} \sum_{C \in W_{n}} m(C)$, hence $m\left(A_{n}\right) \leq n^{-(1+\varepsilon)}$. The Borel-Cantelli Lemma implies that $m$-a.e. $x$ belongs to $A_{n}$ only for finitely many $n$.

Next, for each $n$-cylinder $C$, let $U(C)=\left\{\left.x \in C|| D f^{n}(x)\left|>n^{1+\varepsilon}\right| C\right|^{-1}\right\}$. Write $B_{n}=\bigcup_{C \in \mathcal{C}_{n}} U(C)$. Then

$$
1 \geq \int_{U(C)}\left|D f^{n}(x)\right| d x \geq \frac{n^{1+\varepsilon}}{|C|} m(U(C)),
$$

so that $m\left(B_{n}\right)=\sum_{C \in \mathcal{C}_{n}} m(U(C)) \leq n^{-(1+\varepsilon)} \sum_{C \in \mathcal{C}_{n}}|C|=n^{-(1+\varepsilon)}$. Again the Borel-Cantelli Lemma shows that $m$-a.e. $x$ belongs to $B_{n}$ for only finitely many $n$.

Therefore, for $m$-a.e. $x$, and because $\mu \ll m$ also for $\mu$-a.e. $x$, there exists $n_{0}=n_{0}(x)$ such that $x \notin A_{n} \cup B_{n}$ for all $n \geq n_{0}$. It follows that

$$
\mu\left(C_{n}(x)\right)\left|D f^{n}(x)\right| \leq n^{1+\varepsilon}\left|C_{n}(x)\right| \frac{n^{1+\varepsilon}}{\left|C_{n}(x)\right|} \leq n^{2(1+\varepsilon)} .
$$

The lower bound(s): Here we will apply the Borel-Cantelli Lemma to the invariant measure $\mu$. Let $\widehat{V}_{n}$ be the set of $\widehat{x} \in \widehat{I}$ such that $d\left(\widehat{x}, \partial D_{i}\right) \leq$ $n^{-(l+1)}\left|D_{i}\right|$ for some $i \in \mathbb{N}$. Recall that the densities $h_{k}$ on the levels $D_{k}$ satisfy $h_{k}(y) \leq \delta_{k}\left|y-c_{k}\right|^{1 / l-1}+a_{k-\beta(k)} \delta_{\beta(k)}\left|y-c_{\beta}(k)\right|^{1 / l-1}$. Therefore

$$
\begin{aligned}
\widehat{\mu}\left(\widehat{V}_{n}\right) & \leq \sum_{k}\left(\int_{c_{k}}^{c_{k}+n^{-(l+1)}}+\int_{c_{\beta(k)}-n^{-(l+1)}}^{c_{\beta(k)}}\right) \\
& \leq \sum_{k} \frac{2}{l}\left(\delta_{k}\left|y-c_{k}\right|^{1 / l-1}+a_{k-\beta(k)} \delta_{\beta(k)}\right) n^{-(l+1) / l} \leq C n^{-(l+1) / l}
\end{aligned}
$$

for some constant $C<\infty$. By the Borel-Cantelli Lemma, for $\mu$-a.e. $x$, $f^{n}(\widehat{x}) \in \widehat{V}_{n}$ (for $\widehat{x}=\pi^{-1}(x) \cap D_{2}$ ) for only finitely many $n$. Take such an $x$. Since $f^{n}\left(C_{n}(x)\right)=D_{k}$ if and only if $\widehat{g}(\widehat{x}) \in D_{k}$, we conclude that for $n$ sufficiently large, there is a Koebe space of relative length $n^{-(l+1)}$ around 
$f^{n}(x)$. This gives

$$
\left|D f^{n}(x)\right| \geq\left(\frac{n^{-(l+1)}}{1+n^{-(l+1)}}\right)^{2} \frac{\mid f^{n}\left(C_{n}(x)\right)}{\left|C_{n}(x)\right|} \geq \frac{1}{2 n^{2(l+1)}} \frac{\left|f^{n}\left(C_{n}(x)\right)\right|}{\left|C_{n}(x)\right|} .
$$

It follows that

$$
\mu\left(C_{n}(x)\right)\left|D f^{n}(x)\right| \geq \frac{b}{n^{2(l+1)}}\left|f^{n}\left(C_{n}(x)\right)\right| .
$$

Finally we give a lower bound for $\left|C_{n}(x)\right|$. Take $\widehat{U}_{n}=\bigsqcup\left\{D_{k}|| D_{k} \mid<\right.$ $\left.n^{-(l+1)}\right\}$. Then

$$
\widehat{\mu}\left(\widehat{U}_{n}\right) \leq \sum_{D_{k} \subset \widehat{U}_{n}} \int_{D_{k}} h_{k}(y) d y \leq \frac{n^{-(l+1) / l}}{l} \sum_{k}\left(\delta_{k}+a_{k-\beta(k)} \delta_{\beta(k)}\right) \leq C n^{-(l+1) / l} .
$$

Using Borel-Cantelli again, we find that for $\mu$-a.e. $x, f^{n}\left(C_{n}(x)\right) \leq n^{-(l+1)}$ with at most finitely many exceptions.

3.3. The Central Limit Theorem. We begin with a characterization of the potential $\varphi=\log \left|f^{\prime}\right|-\int \log \left|f^{\prime}\right| d \mu$, which will be useful in order to apply a theorem by Gordin (see below).

Lemma 6. The potential $\varphi=\log \left|f^{\prime}\right|-\int \log \left|f^{\prime}\right| d \mu$ belongs to $L^{2}(\mu)$.

Proof. First observe that there exists $K$ such that $\mu((c-\varepsilon, c+\varepsilon))<K \varepsilon$ for all $\varepsilon$. Indeed, by (5), and analogously to the computations in Lemma 4 ,

$$
\begin{aligned}
\mu((c-\varepsilon, c+\varepsilon)) & \leq C \sum_{k} \int_{c-\varepsilon}^{c+\varepsilon}\left|y-c_{k}\right|^{1 / l-1}\left|D f^{k-1}\left(c_{1}\right)\right|^{-1 / l} d y \\
& \leq 2 C \varepsilon \sum_{k}\left|D f^{k-1}(f(c))\right|^{-1 / l} \int_{0}^{\varepsilon}|y|^{1-1 / l} d y \leq K \varepsilon^{1 / l},
\end{aligned}
$$

similarly to (7). As $\log \left|f^{\prime}\right|=\mathcal{O}(l) \log |x-c|$,

$$
\begin{aligned}
\int\left(\log \left|f^{\prime}\right|\right)^{2} d \mu & \leq \sum_{n} K 2^{(1-n) / l}\left|\mathcal{O}(l) \log 2^{-n}\right|^{2} \\
& \leq 2 K \log 2 \mathcal{O}\left(l^{2}\right) \sum_{n} 2^{-n / l} n^{2}<\infty
\end{aligned}
$$

Let us now prove Theorem 4, which states that the Central Limit Theorem holds for $\varphi$. The condition that the variance is positive is non-trivial, but can, as usual, be reduced to the condition that $\varphi$ is not cohomologous to 0 . Notably, $\sigma_{\mu}^{2}(\varphi)=0$ for the full quadratic map $f: x \mapsto 4 x(1-x)$. The reason is that $f$ is differentiably conjugate to the full tent-map $T$ : $x \mapsto 1-2|x|$. The logarithm of the derivative of the conjugacy $\psi:=\log h^{\prime}$ satisfies $\varphi=\psi-\psi \circ f=0$, which in turn implies that $\sigma_{\mu}^{2}(\varphi)=0$. In this case, the lengths of cylinder sets are too regular, so the fluctuation of return 
time statistics is less than expected. The precise form of the fluctuation in this case follows from the results in [10]. In general, if $\varphi=\psi-\psi \circ f$ has a solution, Gordin's [12] results imply that $\psi=\sum_{j} P^{j}(\varphi) \in L^{1}(\mu)$, where $P$ is the Perron-Frobenius operator. If in addition $\exp (\psi) \in L^{1}(\mu)$, then the integral of $\exp (\psi)$ conjugates $f$ to a tent map. We conjecture that $\sigma_{\mu}^{2}(\varphi)=0$ is very unlikely in the class of maps under consideration.

Proof of Theorem 3. In [7], it was shown that Hölder observables satisfy the Central Limit Theorem. In our case, $\varphi$ is not Hölder, and not even bounded. However $\varphi \in L^{2}(\mu)$ (see Lemma 6), and the singularity of $\varphi$ is localized and of logarithmic order. From the proofs of [34] and [7] combined, it turns out that when $\varphi$ is lifted to an appropriate tower, it satisfies the required Hölder-like property. Let us discuss the construction more precisely.

In [7] a jump transformation $F: \bigcup_{i} \omega_{i} \subset \Omega_{0} \rightarrow \Omega_{0}$ is constructed, where $\Omega_{0}$ is an interval, and $\left\{\omega_{i}\right\}_{i}$ an interval partition of $\Omega_{0}$ such that:

- The Lebesgue measure $m\left(\Omega_{0} \backslash \bigcup_{i} \omega_{i}\right)$ is 0 .

- For each $i, F\left|\omega_{i}=f^{R_{i}}\right| \omega_{i}$ for some $R_{i} \geq 1$, and $F: \omega_{i} \rightarrow \Omega_{0}$ is a diffeomorphism with uniformly (in $i$ ) bounded distortion.

- The inducing times $R_{i}$ are summable:

$$
\sum_{i} R_{i} m\left(\omega_{i}\right)<\infty
$$

If we assume that $\left|D f^{n}\left(c_{1}\right)\right| \geq C n^{\tau}$ for some $\tau>4 l-3$, then the tail satisfies $\sum_{R_{i}>n} m\left(\omega_{i}\right) \leq \mathcal{O}\left(n^{-\alpha}\right)$ for any $\alpha \in\left(3, \frac{\tau-1}{l-1}-1\right)$. Under Hölder conditions, the Central Limit Theorem holds.

In [7], $\Omega_{0}$ is a neighborhood of the critical point, but the same construction is valid if $\Omega_{0}$ is a neighborhood bounded away from $c$. In addition, the construction of $F$ (see the definition of the binding period $p(x)$ in [7]) implies that there is a constant $\kappa>0$ such that

$$
\inf \left\{|c-y| \mid y \in f^{j}\left(\omega_{i}\right)\right\}>\kappa\left|f^{j}\left(\omega_{i}\right)\right|
$$

for all $i$ and $0 \leq j<R_{i}$.

The paper [7] proceeds to invoke [34] for the following tower construction:

$$
\Delta=\bigsqcup_{i} \bigsqcup_{j=0}^{R_{j}-1} \omega_{i, j}
$$

where $\omega_{i, j}=\omega_{i}$ for each $j$. Equip $\Delta$ with Lebesgue measure $\widetilde{m}$ as reference measure: $\widetilde{m}\left|\omega_{i, j}=m\right| \omega_{i}$. By (9), $\widetilde{m}$ is a finite measure. Define a map $\widetilde{f}$ : $\Delta \rightarrow \Delta$ by

$$
\widetilde{f}\left(x \in \omega_{i, j}\right)= \begin{cases}x \in \omega_{i, j+1} & \text { if } j+1<R_{i}, \\ f^{R_{i}}(x) \in \Omega_{0} & \text { if } j+1=R_{i},\end{cases}
$$


where $\Omega_{0}=\bigcup_{i} \omega_{i, 0} \bmod m$. The system $(\Delta, \tilde{f})$ is a countable Markov system, and in view of (9), a standard argument produces an $\tilde{f}$-invariant probability measure $\widetilde{\mu}$ such that, for each $\omega_{i, j}, d \widetilde{\mu} / d m$ is bounded and bounded away from 0 . Define the projection $\pi: \Delta \rightarrow I$ by $\pi\left(x \in \omega_{i, j}\right)=f^{j}(x)$. Lift $\varphi$ to the tower, i.e., take $\widetilde{\varphi} \mid \omega_{i, j}=\varphi \circ f^{j}$. The $f$-invariant measure $\mu$ on $I$ satisfies $\mu=\widetilde{\mu} \circ \pi^{-1}$.

We need a theorem of Gordin [12], cited in [33], which translates in our notation to

TheOREM 6 (Gordin). Suppose $\widetilde{\varphi} \in L^{2}(\widetilde{\mu})$, and

$$
\sum_{n \geq 0} \sqrt{\int\left|\mathbb{E}\left(\widetilde{\varphi} \mid \tilde{f}^{-n} \mathcal{B}\right)\right|^{2} d \widetilde{\mu}}<\infty
$$

where $\mathcal{B}$ is the algebra of Lebesgue measurable sets on $\Delta$, and $\mathbb{E}\left(\widetilde{\varphi} \mid \widetilde{f}^{-n} \mathcal{B}\right)$ is the conditional expectation with respect to $\widetilde{\mu}$. Then $\widetilde{\varphi}$ satisfies the Central Limit Theorem.

Let $\widetilde{h}=d \widetilde{\mu} / d \widetilde{m}$ be the density of $\widetilde{\mu}$. If $U(\widetilde{\varphi}):=\widetilde{\varphi} \circ \tilde{f}$ and $U^{*}$ is its dual operator (with respect to $\widetilde{\mu}$ ), then we can compute (cf. [33]) $U^{* n}(\widetilde{\varphi})=$ $P^{n}(\widetilde{\varphi} \widetilde{h}) / \widetilde{h}$, where $P$ denotes the Perron-Frobenius operator. Furthermore,

$$
\mathbb{E}\left(\widetilde{\varphi} \mid \tilde{f}^{-n} \mathcal{B}\right)(x)=U^{n} U^{n *}(\widetilde{\varphi})(x)=\frac{1}{\widetilde{h} \circ \widetilde{f}^{n}(x)} \sum_{\widetilde{f}^{n}(y)=\widetilde{f}^{n}(x)}\left|\frac{(\widetilde{\varphi} \tilde{h})(y)}{D \widetilde{f}^{n}(y)}\right| .
$$

Therefore

$$
\begin{aligned}
\int_{\Delta}\left|\mathbb{E}\left(\widetilde{\varphi} \mid \tilde{f}^{-n} \mathcal{B}\right)\right|^{2} d \widetilde{\mu} & =\int_{\Delta}\left|U^{n} U^{* n}(\widetilde{\varphi})\right|^{2} d \widetilde{\mu}=\int_{\Delta}\left|U^{* n}(\widetilde{\varphi})\right|^{2} d \widetilde{\mu} \\
& =\int_{\Delta}\left|U^{n} U^{* n}(\widetilde{\varphi}) \cdot \widetilde{\varphi}\right| d \widetilde{\mu}
\end{aligned}
$$

Let $\Delta_{n}:=\pi^{-1}\left(B\left(c ; L^{-n}\right)\right)$ be the lift of the $L^{-n}$-ball around $c$, where $L=$ $\sup \left|f^{\prime}\right|$. If $f^{j}\left(\omega_{i}\right) \cap B\left(c ; L^{-n}\right) \neq \emptyset$, then by $(10),\left|f^{j}\left(\omega_{i}\right)\right| \leq 1 /\left(\kappa L^{n}\right)$, and

$$
\left|f^{j+n}\left(\omega_{i}\right)\right| \leq L^{n-1}\left|f^{j+1}\left(\omega_{i}\right)\right| \leq(\kappa L)^{-1}\left|f^{\prime}(\xi)\right|=\mathcal{O}\left(L^{-n(l-1)}\right) \ll\left|\Omega_{0}\right|
$$

for some $\xi \in f^{j}\left(\omega_{i}\right)$. Therefore $R_{i}>j+n$. It follows that if $y \in \omega_{i, j}$, then $\tilde{f}^{-n} \circ \tilde{f}^{n}(y)=\{y\}$, and also

$$
\frac{\widetilde{h}(y)}{\left|D \widetilde{f}^{n}(y)\right| \widetilde{h}^{n} \widetilde{f}^{n}(y)}=1
$$

Hence for such $y$, the integrand above is $\left|U^{n} U^{* n}(\widetilde{\varphi}) \cdot \widetilde{\varphi}\right|(y)=|\widetilde{\varphi}(y)|^{2}$.

Next let $\Delta_{n}^{\prime}:=\pi^{-1}\left(B\left(c ; n^{-5}\right) \backslash B\left(c ; L^{-n}\right)\right)$. Then $|\widetilde{\varphi}| \leq \mathcal{O}(n(l-1) \log L)$ on $\Delta_{n}^{\prime}$, whereas $|\widetilde{\varphi}| \leq \mathcal{O}(5(l-1)) \log n$ on $\pi^{-1}\left(I \backslash B\left(c ; n^{-5}\right)\right)$. If $y \in \Delta_{n}^{\prime}$, 
then $\tilde{f}^{-n} \circ \tilde{f}^{n}(y) \cap \Delta_{n}=\emptyset$. Therefore

$$
\begin{aligned}
\int_{\Delta_{n}^{\prime} \backslash \Delta_{n}}\left|U^{n} U^{* n}(\widetilde{\varphi}) \cdot \widetilde{\varphi}(x)\right| d \widetilde{\mu} & =\int_{\Delta_{n}^{\prime} \backslash \Delta_{n}} \frac{\widetilde{\varphi}(x)}{\widetilde{h} \circ \widetilde{f}^{n}(x)} \sum_{\widetilde{f}^{n}(y)=\widetilde{f}^{n}(x)}\left|\frac{\widetilde{\varphi}(y)}{D \widetilde{f}(y)}\right| d \widetilde{\mu} \\
& \leq \int_{\Delta_{n}^{\prime} \backslash \Delta_{n}} \mathcal{O}\left(n^{2}\right) d \widetilde{\mu} .
\end{aligned}
$$

Combining these estimates, we can continue (11) as

$$
\begin{aligned}
& \leq \int_{\Delta_{n}}|\widetilde{\varphi}(y)|^{2} d \widetilde{\mu}+\int_{\Delta_{n}^{\prime} \backslash \Delta_{n}} \mathcal{O}\left(n^{2}\right) d \widetilde{\mu}+\mathcal{O}(\log n) \int_{\Delta}\left|U^{n} U^{* n}(\widetilde{\varphi})\right| d \widetilde{\mu} \\
& \leq \mathcal{O}\left(L^{-n} n^{2}\right)+\mathcal{O}\left(n^{-3}\right)+\mathcal{O}(\log n) \int_{\Delta}\left|U^{* n}(\widetilde{\varphi})\right| d \widetilde{\mu} \\
& =\mathcal{O}\left(L^{-n} n^{2}\right)+\mathcal{O}\left(n^{-3}\right)+\mathcal{O}(\log n) \int_{\Delta}\left|P^{n}(\widetilde{\varphi} \tilde{h})\right| d \widetilde{m} .
\end{aligned}
$$

We next prove that $\tilde{\varphi} \widetilde{h}$ has sufficient Hölder properties. We chose $\Omega_{0}$ bounded away from $c$, so $\varphi$ is $C^{2}$ on $\Omega_{0}$. Given $x, y \in \omega_{i, j}$, the separation time $s(x, y)$, as defined in [34, Section 1.1], counts the minimal number of returns to $\Omega_{0}$ before $x$ and $y$ belong to different partition elements $\omega_{k}$. Because $F$ is expanding and $f^{R_{i}-j}: f^{j}\left(\omega_{i}\right) \rightarrow \Omega_{0}$ has bounded distortion, it is not hard to check that $|x-y| /\left|\omega_{i, j}\right|$ is exponentially small in $s(x, y)$ (see also [7, Lemma 4.5]).

Write

$$
\widetilde{\varphi} \widetilde{h}=C\left(\frac{d \lambda}{d \widetilde{m}}-\frac{d \lambda^{\prime}}{d \widetilde{m}}\right)
$$

as the scaled difference of two probability densities, for example

$$
\frac{d \lambda}{d \widetilde{m}}=\frac{1}{C}(1+\max \{\widetilde{\varphi} \tilde{h}, 0\}) \quad \text { and } \quad \frac{d \lambda^{\prime}}{d \widetilde{m}}=\frac{1}{C}(1-\min \{\widetilde{\varphi} \widetilde{h}, 0\}) .
$$

Here $d \lambda^{\prime} / d \widetilde{m}$ has the logarithmic singularity, and $C>0$ is a normalizing constant. On each interval $\omega_{i, j}, \widetilde{h}(x)$ is Hölder continuous, bounded and bounded away from 0 . Therefore, there exists $\beta \in(0,1)$ such that for all $x, y \in f^{j}\left(\omega_{i}\right)$,

$$
\begin{aligned}
\left|\frac{\frac{d \lambda^{\prime}}{d \tilde{m}}(x)}{\frac{d \lambda^{\prime}}{d \widetilde{m}}(y)}-1\right| & \leq \mathcal{O}(1)\left|\frac{\log |x-c|}{\log |y-c|}-1\right| \leq \mathcal{O}(1) \frac{|x-y|}{|y-c|} \\
& \leq \mathcal{O}\left(\frac{1}{\kappa}\right) \frac{|x-y|}{\left|f^{j}\left(\omega_{i}\right)\right|} \leq \text { const } \beta^{s(x, y)} .
\end{aligned}
$$

The estimates for $d \lambda / d \widetilde{m}$ are similar, and give the same upper bound. Hence both $d \lambda / d \widetilde{m}$ and $d \lambda^{\prime} / d \widetilde{m}$ belong to $C_{\beta}^{+}(\Delta)$ as in [34]. Following the [34] 
argument, we obtain for the correlations

$$
\int_{\Delta}\left|P^{n}(\widetilde{\varphi} \tilde{h})\right| d \widetilde{m}=\left|\tilde{f}_{*}^{n} \lambda-\widetilde{f}_{*}^{n} \lambda^{\prime}\right|(\Delta) \leq \mathcal{O}\left(n^{-(\alpha-1)}\right)
$$

for the $\alpha>3$ as above. Therefore $\sqrt{\int_{\Delta}\left|\mathbb{E}\left(\widetilde{\varphi} \mid \tilde{f}^{-n} \mathcal{B}\right)\right|^{2} d \widetilde{\mu}}$ is indeed summable, and by Gordin's Theorem, $\widetilde{\varphi}$ satisfies the Central Limit Theorem. The same is true for the original observable $\varphi$.

\section{References}

[1] M. Abadi, Exponential approximation for hitting times in mixing processes, Math. Phys. Electron. J. 7 (2001), paper 2, 19 pp.

[2] L. Barreira and B. Saussol, Hausdorff dimension of measures via Poincaré recurrence, Comm. Math. Phys. 219 (2001) 443-463.

[3] A. Boubakri, Distribution asymptotique des temps d'entrée pour une classe d'applications unimodales de l'intervalle, thèse, Univ. de Dijon, 1996.

[4] H. Bruin, Combinatorics of the kneading map, Internat. J. Bifur. Chaos 5 (1997), $1339-1349$.

[5] -, Induced maps, Markov extensions and invariant measures in one-dimensional dynamics, Comm. Math. Phys. 168 (1995), 571-580.

[6] - Invariant measures of interval maps, Ph.D. thesis, Delft, 1994.

[7] H. Bruin, S. Luzzatto and S. van Strien, Decay of correlation in one-dimensional dynamics, Ann. Sci. École Norm. Sup., to appear.

[8] H. Bruin, B. Saussol, S. Troubetzkoy and S. Vaienti, Return time statistics via inducing, Ergodic Theory Dynam. Systems, to appear.

[9] P. Collet, Statistics of closest return for some non-uniformly hyperbolic systems, ibid. 21 (2001), 401-420.

[10] P. Collet, A. Galves and B. Schmitt, Repetition times for Gibbsian sources, Nonlinearity 12 (1999), 1225-1237.

[11] A. Galves and B. Schmitt, Inequalities for hitting times in mixing dynamical systems, Random Comput. Dynam. 5 (1997), 337-348.

[12] M. I. Gordin, The central limit theorem for stationary processes, Soviet Math. Dokl. 10 (1969), 1174-1176.

[13] N. Haydn, J. Luevano, G. Mantica and S. Vaienti, Multifractal properties of return time statistics, Phys. Rev. Lett. 38 (2002), 224502-1/4.

[14] N. Haydn and S. Vaienti, The limiting distribution and error terms for return times of dynamical systems, preprint, 2001.

[15] - - - The central limit theorem for $(\varphi, f)$-mixing maps, preprint, 2001.

[16] M. Hirata, B. Saussol and S. Vaienti, Statistics of return times: a general framework and new applications, Comm. Math. Phys. 206 (1999), 33-55.

[17] G. Keller, Lifting measures to Markov extensions, Monatsh. Math. 108 (1989), 183-200.

[18] - Exponents, attractors and Hopf decompositions for interval maps, Ergodic Theory Dynam. Systems 10 (1990), 717-744.

[19] G. Keller and T. Nowicki, Spectral theory, zeta functions and the distribution of periodic points for Collet-Eckmann maps, Comm. Math. Phys. 149 (1992), 31-69.

[20] I. Kontoyiannis, Asymptotic recurrence and waiting times for stationary processes, J. Theoret. Probab. 11 (1998), 795-811. 
[21] O. Kozlovski, Getting rid of the negative Schwarzian derivative condition, Ann. of Math. 152 (2000), 743-762.

[22] F. Ledrappier, Some properties of absolutely continuous invariant measures on an interval, Ergodic Theory Dynam. Systems 1 (1981), 77-93.

[23] M. Martens, Distortion results and invariant Cantor sets, Ergodic Theory Dynam. Systems 14 (1994), 331-349.

[24] W. de Melo and S. van Strien, One-Dimensional Dynamics, Springer, 1993.

[25] T. Nowicki, Some dynamical properties of S-unimodal maps, Fund. Math. 142 (1993), 45-57.

[26] T. Nowicki and D. Sands, Non-uniform hyperbolicity and universal bounds for $S$ unimodal maps, Invent. Math. 132 (1998), 633-680.

[27] T. Nowicki and S. van Strien, Invariant measures exist under a summability condition, ibid. 105 (1991), 123-136.

[28] D. Ornstein and B. Weiss, Entropy and data compression, IEEE Trans. Inform. Theory 39 (1993), 78-83.

[29] F. Paccaut, Statistics of return times for weighted maps of the interval, Ann. Inst. H. Poincaré Probab. Statist. 36 (2000), 339-366.

[30] B. Saussol, Exponential statistics of return times, Nonlinearity 14 (2001), 179-191.

[31] B. Saussol, S. Troubetzkoy and S. Vaienti, Recurrence, dimension and Lyapunov exponent, J. Statist. Phys., to appear.

[32] B. Szewc, The Perron-Frobenius operator in spaces of smooth functions on an interval, Ergodic Theory Dynam. Systems 4 (1984), 613-641.

[33] L.-S. Young, Statistical properties of dynamical systems with some hyperbolicity, Ann. of Math. 147 (1998), 585-650.

[34] —, Recurrence times and rates of mixing, Israel J. Math. 110 (1999), 153-188.

Department of Mathematics

University of Groningen

P.O. Box 800

9700 AV Groningen, The Netherlands

E-mail: bruin@math.rug.nl

URL: http://www.math.rug.nl/ ^bruin
PHYMAT

Université de Toulon

Centre de Physique Théorique et FRUMAM

Fédération de Recherche des Unités de Mathématiques de Marseille CNRS Luminy, Case 907

F-13288 Marseille Cedex 9, France

E-mail: vaienti@cpt.univ-mrs.fr URL: http://www.cpt.univ-mrs.fr/

Received 3 June 2002;

in revised form 27 August 2002 